\title{
Wave packet analysis and break-up length calculations for an accelerating planar liquid jet
}

\author{
M. R. Turner ${ }^{\text {a } 12}$, J. J. Healey ${ }^{\text {b }, ~ S . ~ S . ~ S a z h i n ~}{ }^{\text {a }}$ \& R. Piazzesi ${ }^{\text {a }} 3$ \\ ${ }^{a}$ Sir Harry Ricardo Laboratories, \\ School of Computing, Engineering and Mathematics, University of Brighton, \\ Lewes Road, Brighton, East Sussex BN2 4 GJ UK \\ ${ }^{\mathrm{b}}$ Department of Mathematics, Keele University, Keele, Staffs ST5 5BG UK
}

\begin{abstract}
This paper examines the process of transition to turbulence within an accelerating planar liquid jet. By calculating the propagation and spatial evolution of disturbance wave packets generated at a nozzle where the jet emerges, we are able to estimate break-up lengths and break-up times for different magnitudes of acceleration and different liquid to air density ratios. This study uses a basic jet velocity profile which has shear layers in both the air and the liquid either side of the fluid interface. The shear layers are constructed as functions of velocity which behave in line with our CFD simulations of injecting Diesel jets. The non-dimensional velocity of the jet along the jet centre-line axis is assumed to take the form $V(t)=\tanh (a t)$ where the parameter $a$ determines the magnitude of the acceleration. We compare the fully unsteady results obtained by solving the unsteady Rayleigh equation, to those of a quasi-steady jet to determine when the unsteady effects are significant, and if the jet can be regarded as quasi-steady in typical operating conditions for Diesel engines.
\end{abstract}

For a heavy fluid injecting into a lighter fluid (density ratio $\rho_{\text {air }} / \rho_{\text {jet }}=q<1$ ) it is found that unsteady effects are mainly significant at early injection times where the jet velocity profile is changing fastest. When the shear layers in the jet thin with time, the unsteady effects cause the growth rate of the wave packet to be smaller than the corresponding quasi-steady jet, while for thickening shear layers the unsteady growth rate is larger than that of the quasi-steady jet. For large accelerations (large $a$ ) the unsteady effect remains at later times but its effect on the growth rate of the wave packet decreases as the time of injection increases. As

1 Corresponding author: M.Turner@surrey.ac.uk

2 Present address: Department of Mathematics, University of Surrey, Guildford, Surrey GU2 7XH UK

3 Present address: ANSYS UK, Ltd., 97 Milton Park, Abingdon, Oxfordshire OX14 4RY UK 
the rate of acceleration is reduced, the range of velocity values that the jet can be considered as quasi-steady increases until eventually the whole jet can be considered quasi-steady. For a homogeneous jet $(q=1)$ the range of values of $a$ for which the jet can be considered completely quasi-steady increases to larger values of $a$.

Finally we investigate approximating the wave packet break-up length calculations with a method which follows the most unstable disturbance wave as the jet accelerates. This approach is similar to that used in CFD simulations as it greatly reduces computational time. We investigate whether or not this is a good approximation for the parameter values typical for Diesel engines.

\section{Introduction}

Within a Diesel engine there occur many processes which are fundamental to the running of the engine $[1,2]$. These processes include the injection of the fuel, the break-up of the resulting jet into droplets and the heating and evaporation of these droplets to name but a few [3]. Each of these processes needs to be accurately modeled so that they can be combined in CFD simulations. In the present study we concentrate on the injection process. The liquid fuel is injected into the combustion chamber through an injector (which can have multiple holes) where it breaks up into liquid sheets, ligaments and droplets before evaporating and burning up in the autoignition and combustion processes. From this process, one important piece of information is the 'break-up length' of the jet, which is the distance from the nozzle to the point at which liquid sheets, ligaments and droplets begin to form [4,5]. The calculation of this length and the overall modeling of this process is very important to the modelling of the processes in Diesel engines using CFD codes [6]. Most of the injection models used to date determine the break-up length using the stability characteristics of steady liquid jets [7-10]. However the liquid jet in this injection process is not steady and is undergoing acceleration at the initial stage. Some authors believe that this acceleration is key to determining the differences that occur between the experimental observations and numerical CFD simulations [6], which essentially use quasi-steady approximations to model break-up, where the CFD simulations underestimate the observed break-up length. The purpose of this paper is to clarify whether or not this statement is true. The same steady stability characteristics are also used to determine the penetration length of fuel sprays [7], which is the maximum distance at which droplets are observed when a spray is injected into a combustion chamber. In a spray with a coherent jet at the nozzle the penetration length is related to the break-up length and so understanding the role of unsteadiness in jets will also help to better understand why these penetration results too are underestimated by the simulations by as much as $50 \%$ [6]. In this paper we study the effect of acceleration on liquid jets to determine 
when the acceleration effects significantly affect the break-up length and how we might be able to incorporate this effect into CFD simulations. While the jets in Diesel engines are approximately axisymmetric, we consider planar jets in this study as these allow us to simplify the analysis. Our main objective is to generate a qualitative understanding of the effect of acceleration, not to generate quantitative agreement with experiments at this stage. The stability and break-up properties of both steady axisymmetric and planar jets have been studied both experimentally and theoretically in the past, and for a good overview of these studies the reader is directed to the introduction of [11]. This planar approximation also means that we are neglecting the effect of swirl on the jet break-up length. Experiments have shown that swirl reduces the break-up length in both viscous $[12,13]$ and more inviscid jets [14].

A planar jet consists of two parallel shear layers where the vorticity at each layer is equal and opposite. The stability properties of such jets are found by performing a linear stability analysis about a basic velocity profile $U(y)$ where $y$ is a coordinate normal to the jet axis. By looking for a traveling wave solution of the linearized Navier-Stokes equations of the form $\hat{v}(x, y, t)=$ $\tilde{v}(y) \exp [i(\alpha x-\omega t)]$, in the absence of viscosity, we arrive at the Rayleigh equation

$$
(\alpha U-\omega)\left(\frac{d^{2} \tilde{v}}{d y^{2}}-\alpha^{2} \tilde{v}\right)-\alpha \frac{d^{2} U}{d y^{2}} \tilde{v}=0,
$$

where $\tilde{v}(y)$ is the velocity component normal to the jet axis (in the $y$-direction), $t$ is time, $\alpha$ is the streamwise wavenumber and $\omega$ is the complex angular frequency; see [15]. Here we neglect the effect of viscosity as experimental studies have shown that the Kelvin-Helmholtz/Rayleigh instability is the dominant instability in the break-up of jets [16]. Thus, as typical Reynolds numbers for Diesel jets are larger than $10^{3}$, viscosity can be neglected in this problem, as it can be in channel flows [17]. The effects of viscosity are implicitly taken into account in this study via the form of the velocity profile $U$.

By performing a temporal stability analysis on this equation, the range of real $\alpha$ values for which the individual disturbance waves grow $\left(\operatorname{Im}(\omega)=\omega_{i}>0\right)$ can be determined. This range of values, as well as the magnitude of $\omega_{i}$, depends upon the thickness of the shear layers at the edge of the jet, and the magnitude of the surface tension $[18,19]$. By forming the superposition of all the real wavenumbers $\alpha$, the spatio-temporal evolution of a wave packet is found. The stability properties of this wave packet for a steady jet have been well studied by $[20,21]$ and [22] for example, where the latter have shown that the break-up length of a steady jet depends upon the exact form of the velocity profile $U(y)$. It was found from their CFD simulations for an axisymmetric jet that steady jets at high velocities, have a thinner shear layer in the liquid close to the nozzle than steady jets at lower velocities. These simulations also showed that the higher velocity jets have a larger ratio of the fluid velocity at the fluid interface to the maximum centre-line velocity, than the slower jets. 
The break-up length of the jet as a function of its velocity was shown to agree qualitatively with experimental results. In this paper we use a similar profile, found from a CFD simulation, to model accelerating jets.

Steady flows can be classed as stable, convectively unstable or absolutely unstable [23]. Unsteady flows cannot be labeled as easily because the growth rate $\omega_{i}$ and phase speed $c_{r}=\omega_{r} / \alpha$ of each wave of (1.1) are now functions of time and so the flow could go through different states as time evolves. This has been addressed by describing instantaneous stability results, where some measure of the flow is examined to see if it is growing or decaying at a particular time $[24,25]$. We note here that (1.1) does not strictly apply to an unsteady flow, but it should be a valid approximation when the magnitude of the acceleration is weak enough. In the present work we are less concerned with classifying the flow as stable or unstable etc, but more interested in finding the absolute amplitude of a wave packet as it evolves. Furthermore we will go beyond instantaneous stability results and solve the full unsteady disturbance equations. Unsteady flows that are periodic in time have been well studied in the literature by using Floquet theory which investigates whether there is net growth or decay over a complete period of the basic flow. These flows can be solved by well established methods [26-29]. However, the stability of nonperiodic unsteady flows has not been studied to such an extent and yet they have many practical applications, such as the fuel injector problem discussed in this paper.

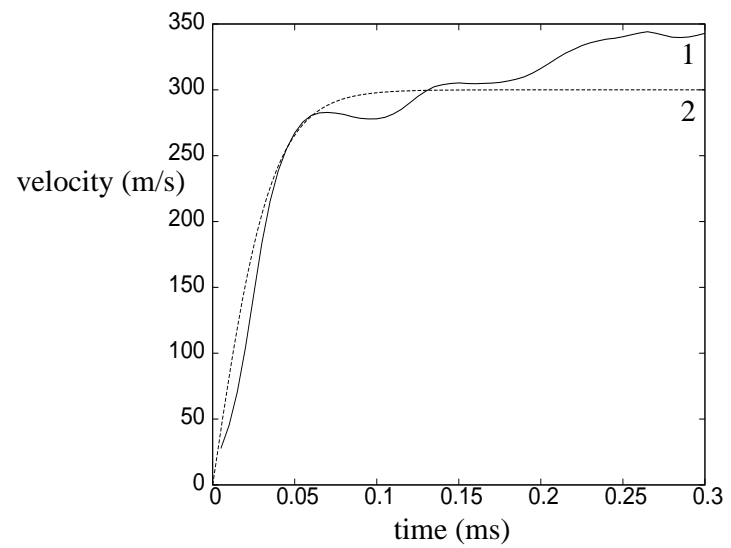

Fig. 1. Plot of the injection velocity as a function of time (curve 1) for a Diesel fuel jet observed experimentally [30], and an approximation to the injection process using the hyperbolic tangent function (curve 2). For this experiment the ratio of the air density in the chamber to the liquid fuel density was $q=\rho_{2} / \rho_{1} \approx 1 / 12$.

Figure 1 shows an example of a typical injection velocity (curve 1), measured experimentally, as a function of time [30]. Although the maximum velocity value and initial rate of acceleration may change, the structure of this profile is typical of Diesel jets. The injection velocity has an initial region where the fluid is rapidly accelerated, after which there is a slow oscillatory injection region, but this is not of interest to us here because initial jet break-up has 
occurred before this region is reached. In this paper we approximate the rapid acceleration via a hyperbolic tangent function as shown by curve 2 in figure 1. Using this approximation, we can examine how disturbances generated at different times at the nozzle, propagate downstream and eventually lead to the jet breaking up into liquid sheets, ligaments and droplets. We assume this break up happens when the disturbance reaches some threshold amplitude where non-linear effects become important. Disturbances which leave the nozzle at early times will be subject to high levels of acceleration, ones which leave at late times will experience an approximately steady jet, and those in between will see moderate acceleration effects. The purpose of this study is to investigate how this acceleration affects the break-up length of the jet, and find out if the magnitude of the acceleration for Diesel jets is large enough so that the break-up length of the unsteady jet is significantly longer than that of the quasi-steady jet.

We examine this problem by using a particular jet velocity profile $U(y, t)$ that captures essential features predicted by CFD simulations. The velocity profile we use approximates the CFD profiles obtained by taking into account the effects of viscosity and has a shear layer either side of the fluid interface. The profile has the properties that the shear layer in the jet thins as the jet velocity increases, while the ratio of the velocity at the interface to the maximum jet velocity also increases with velocity. The actual form of the velocity profile depends upon the geometry of the nozzle (length to diameter ratio, contraction ratio etc) but we expect the results of this paper to be qualitatively similar for many nozzle types. Although our motivation for this work comes from transient Diesel jets, we study the problem in more generality. Therefore one parameter of interest is $q=\rho_{2} / \rho_{1}$, where $\rho_{1}$ is the density of the jet and $\rho_{2}$ is the density of the surrounding fluid. We consider a value of $q$ typical to that in Diesel jet experiments, and we also consider the homogeneous case $q=1$. The case $q=1$ is considered as a starting point for the numerical analysis as the problem is simplified when no fluid interface is present.

The present paper is laid out as follows. In $\S 2$ we formulate the problem, discuss how the break-up lengths will be calculated and present the numerical scheme for solving the resulting partial differential equation. In $\S 3$ we present the results of both the unsteady and quasi-steady simulations for the density ratios $q=1 / 35$ (typical for Diesel engine-like conditions) and $q=1$. We also investigate when the growth rate for the unsteady calculations is considerably different to that of the quasi-steady calculation. Finally we investigate an approximate jet break-up method which only follows the evolution of the fastest growing wave and compare these results with the wave packet calculations. Our concluding remarks and discussion are given in $\S 4$. 


\section{Formulation}

In this paper we consider a two-dimensional planar jet which is orientated along the $x^{*}$-axis in the dimensional $\left(x^{*}, y^{*}\right)$ plane with a dimensional velocity profile $U^{*}\left(y^{*}, t^{*}\right)$, emerging from a nozzle of thickness $2 L^{*}$ centred at $\left(x^{*}, y^{*}\right)=$ $(0,0)$. As $t \rightarrow \infty$ the maximum velocity of the jet levels off and we define our reference velocity to be $U_{0}^{*}(0, \infty)$ (see curve 2 of figure 1 ). By using $L^{*}$ and $U_{0}^{*}$ as a reference length and velocity respectively, we introduce non-dimensional variables $(x, y)=\left(L^{*}\right)^{-1}\left(x^{*}, y^{*}\right)$ such that the jet has thickness 2 and a nondimensional velocity profile $U(y, t)$. The fluid within the jet has density $\rho_{1}$ and lies between an outer fluid of density $\rho_{2}$. We denote the jet centre-line velocity $U(y=0, t)$, as $V(t)$, and so we have the condition $V(t) \rightarrow 1$ as $t \rightarrow \infty$.

In this study we assume that the jet does not spread significantly in the $y$-direction as it moves along the $x$-axis in the region of interest to us. Therefore, we consider the basic velocity profile $\mathbf{u}=U(y, t) \mathbf{i}$ as a function of the coordinate normal to the jet and time only, where $\mathbf{i}$ is the unit vector in the streamwise direction. This assumption holds in the vicinity of the nozzle where the jet spreads slowly in space. A typical velocity profile for this type of jet problem will have a shear layer in each fluid near the edge of the jet, and an example of such profiles is given in figure 2 . The velocity profiles in

(a)

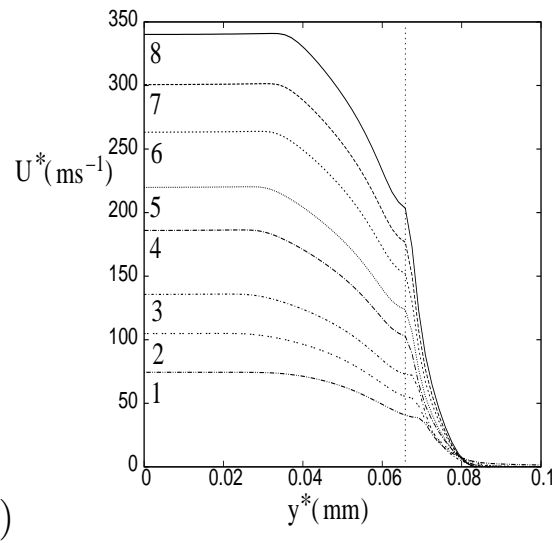

(b)

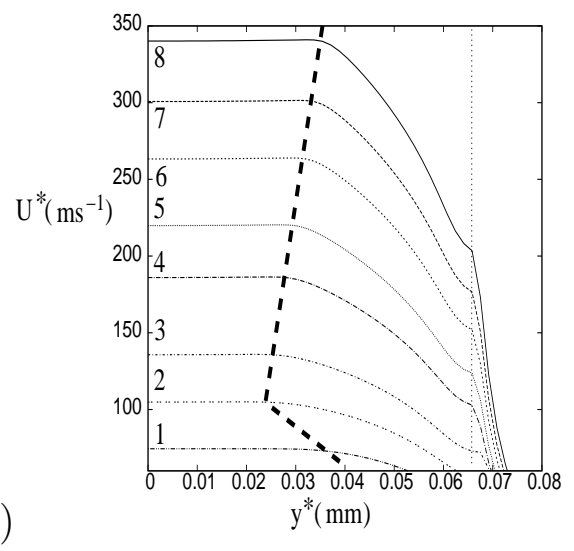

Fig. 2. A selection of (a) velocity profiles from the CFD simulations taken at $0.1 \mathrm{~mm}$ from the nozzle for times $t^{*}=0.035 \mathrm{~ms}, 0.04 \mathrm{~ms}, 0.045 \mathrm{~ms}, 0.055 \mathrm{~ms}, 0.07 \mathrm{~ms}, 0.1 \mathrm{~ms}, 0.175 \mathrm{~ms}$ and $0.3 \mathrm{~ms}$ after injection. These are numbered $1-8$ respectively and $U^{*} / U_{0}^{*}=0.22,0.31,0.40,0.55,0.72,0.77,0.88$ and 1 for these results respectively. The fluid interface is denoted by the vertical dotted line, except for results $1-3$ where the interface lies just to the right of this line due to the fuel bulging out of the nozzle initially. In this simulation $\rho_{1}=700 \mathrm{kgm}^{-3}$, the pressure inside the cylinder is $4 \mathrm{MPa}$ and the temperatures of the fuel and the air are $430 \mathrm{~K}$ and $788 \mathrm{~K}$ respectively. Also, $q=0.0253 \approx 1 / 40$ and the nozzle radius is $0.0675 \mathrm{~mm}$. Panel (b) shows a close up of these profiles showing the approximate edge of the shear layer in the jet. 
figure 2 are calculated for an accelerating, axisymmetric Diesel jet injecting into static air. These profiles were generated using the CFD package ANSYS ${ }^{\circledR}$ FLUENT ${ }^{\circledR}$, where the boundary condition for the mass flow rate of fluid in the nozzle is taken from an in house experiment, see figure 3(a) [31]. The profiles show the evolution of the velocity profile during the injection process and show the slight thinning of the shear layer in the jet as the jet velocity increases beyond about $110 \mathrm{~ms}^{-1}$.

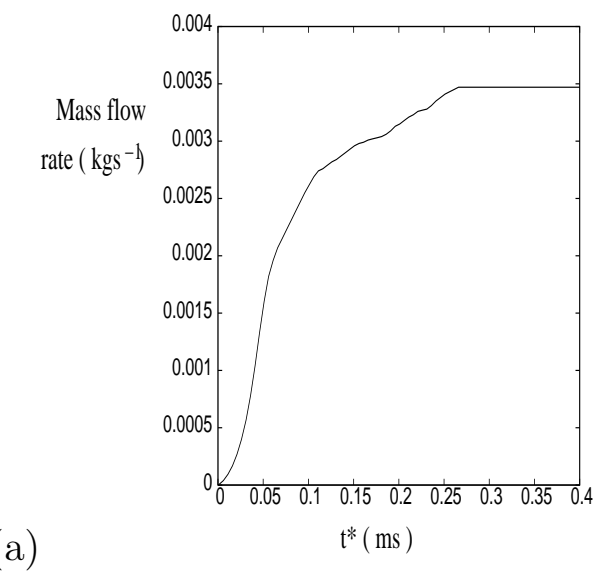

(b)

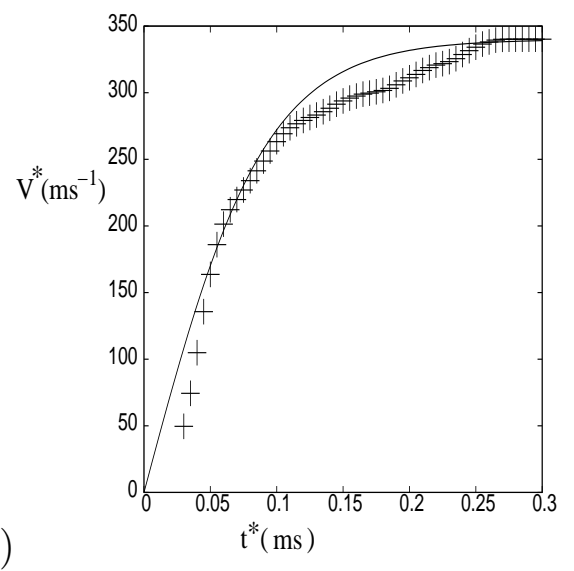

Fig. 3. Plot of (a) the mass flow rate as a function of $t^{*}$, measured by [31] and used as the nozzle boundary condition in the CFD simulations. The crosses in panel (b) show the centre-line velocity of the jet as a function of $t^{*}$ from the CFD simulations at $0.1 \mathrm{~mm}$ from the exit of the nozzle, and the solid line is the approximation $V^{*}=340 \tanh \left(11000 t^{*}\right)$. Here $q=0.0253 \approx 1 / 40$.

The velocity profiles in figure 2 are calculated using the Eulerian multiphase model. In this model a momentum equation for each fluid phase is solved for, giving the respective velocity field. Since there exist large velocity differences between the two phases, this approach allows us to overcome the limitations of the shared velocity and temperature formulation of the Volume of Fluid (VOF) model, which can affect the fluid velocities computed across the interface. We consider the two fluids to be immiscible and the Geo-Reconstruction sharpening scheme $[32,33]$ is used to construct the free surface. The computational domain is a closed cylinder $80 \mathrm{~mm}$ in length and $25 \mathrm{~mm}$ in the radial direction which was chosen to approximate the cylinder of an engine in the experimental facility at the University of Brighton. The nozzle is approximated by a cylindrical channel of $1.08 \mathrm{~mm} \times 0.135 \mathrm{~mm}$ (axial $\times$ radial directions) and is located at the centre of the main cylinder edge. The computational domain is covered by a structured mesh of approximately 82000 nodes which is refined inside the nozzle and in a $0.5 \mathrm{~mm} \times 0.3 \mathrm{~mm}$ region immediately outside the nozzle. A coarser and unstructured mesh is used outside this region and a time step of $\delta_{t^{*}}=5 \times 10^{-8} \mathrm{~s}$ is employed. A standard $\kappa-\epsilon$ turbulent model for both fluids is used, and initially the air in the chamber is considered at rest with a temperature of $755 \mathrm{~K}$ and a pressure of $4 \mathrm{MPa}$. The fuel is injected into the 
cylinder through the nozzle at the constant temperature of $430 \mathrm{~K}$, assuming an adiabatic condition on the walls and applying a mass flow rate boundary condition, given by figure 3(a), at the nozzle inlet surface. This produces a non-uniform velocity profile as the fuel enters the main cylinder. A check of the dependency of the results on the numerical grid was also carried out and the results were found to agree within a few percent.

The mass flow rate in figure 3(a) has been modified so that it levels off once the initial acceleration of the jet has been completed at around $t^{*}=2.5 \times 10^{-4} \mathrm{~s}$. Beyond this time the jet reaches a steady state, although from $t^{*}=2.5 \times$ $10^{-4} \mathrm{~s}$ onwards, the changes in the velocity profiles are small. These velocity profiles are generated assuming that the jet is axisymmetric, but we expect qualitatively similar results for a planar jet, so we use these results to motivate the velocity profiles used in this study. In fact, the experimental results of [11] show planar jet velocity profiles which have a similar appearance to those shown here, although they do not determine the structure of the velocity profile in the outer fluid as we do in our CFD simulations. Figure 3(b) shows the centre-line velocity as a function of time along with an approximation using a hyperbolic tangent function. The profiles in figure 2 are taken at $0.1 \mathrm{~mm}$ from the nozzle exit and the nozzle is assumed to be full of fluid for all times to best model the flow in a Diesel injector where the nozzle fills with fluid as the injector needle is lifted. These profiles were approximated by the piecewise linear profile, shown in figure 4, by [22], which was chosen to facilitate an analytical treatment of the steady jet. In the present work we use smoothed versions of this profile which allows for a numerical treatment of the fully unsteady problem.

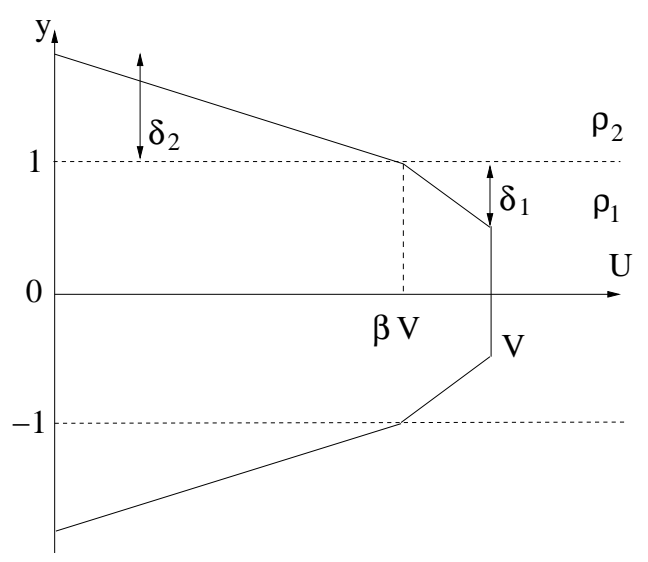

Fig. 4. Plot of the piecewise velocity profile studied in [22] where the thickness of the liquid jet is 2 . The density of the liquid layer is $\rho_{1}$ and has a shear layer width of $\delta_{1}$ while the air density is $\rho_{2}$ and has a shear layer thickness $\delta_{2}$. The parameter $\beta \in[0,1]$ defines the jet velocity at the fluid interface.

The piecewise linear profile shown in figure 4 is first generalized into the following unsteady profile by allowing $\delta_{1}, \delta_{2}, \beta$ and $V$ to become functions of 
time:

$$
U(y, t)= \begin{cases}0 & y>1+\delta_{2}(t) \\ -\frac{\beta(t) V(t)}{\delta_{2}(t)}\left(y-1-\delta_{2}(t)\right) & 1+\delta_{2}(t)>y>1 \\ V(t)-\frac{(1-\beta(t)) V(t)}{\delta_{1}(t)}\left(y-1+\delta_{1}(t)\right) & 1>y>1-\delta_{1}(t) \\ V(t) & 1-\delta_{1}(t)>y>0\end{cases}
$$

where $\delta_{1}(t)$ is the thickness of the shear layer in the jet (fluid 1), $\delta_{2}(t)$ is the shear layer thickness in the outer fluid (fluid 2) and $\beta(t)$ gives the ratio of the fluid velocity at the interface to the maximum centre-line velocity of the jet. The profile (2.1) is then smoothed off to give the velocity profile used in this paper

$U(y, t)=\frac{V(t)}{2}+\frac{\Delta V(t)}{2}\left(\frac{(1-\beta(t))}{\delta_{1}(t)} \ln \left\{\frac{\cosh \left(\frac{y-1}{\Delta}\right)}{\cosh \left(\frac{y-1+\delta_{1}(t)}{\Delta}\right)}\right\}+\frac{\beta(t)}{\delta_{2}(t)} \ln \left\{\frac{\cosh \left(\frac{y-1-\delta_{2}(t)}{\Delta}\right)}{\cosh \left(\frac{y-1}{\Delta}\right)}\right\}\right)$

where $\Delta$ is a smoothing parameter. This profile is obtained by replacing the step functions in $d U / d y$ by hyperbolic tangent functions and integrating the result with respect to $y$, a method used previously in $\S 5.2$ of [34]. A plot of the velocity profile with $\delta_{1}(t)=\delta_{2}(t)=0.4, \beta(t)=0.2$ and $V(t)=1$ for various values of $\Delta$ is shown in figure 5 . We note that as $\Delta \rightarrow 0$ the profiles become more like the piecewise linear profiles, hence we would expect the smooth and piecewise linear profiles to have similar properties in this limit. In $\S 3$ we consider the profile $(2.2)$ with $\Delta=0.025$.

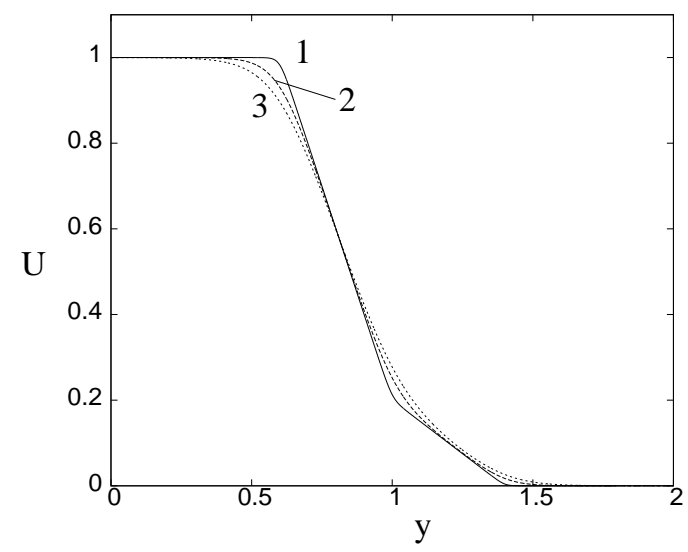

Fig. 5. Plot of the velocity profile (2.2) with $\delta_{1}(t)=\delta_{2}(t)=0.4, \beta(t)=0.2$ and $V(t)=1$ for $\Delta=0.025,0.1$ and 0.15 numbered 1 to 3 respectively.

The stability of profile (2.2) to linear inviscid disturbances is found by linearizing the incompressible non-dimensionalized two-dimensional Euler equations 


$$
\begin{aligned}
\frac{\partial \hat{\mathbf{u}}}{\partial t}+\hat{\mathbf{u}} \cdot \nabla \hat{\mathbf{u}} & =-\nabla \hat{p}, \\
\nabla \cdot \hat{\mathbf{u}} & =0
\end{aligned}
$$

where $\hat{\mathbf{u}}=(\hat{u}, \hat{v})$ is the velocity vector. The work in this paper will also hold for flat jets of a finite thickness, because the Squire transformation will convert the 3D analysis into the below 2D analysis [35]. We introduce velocity and pressure fluctuations of the form

$$
(\hat{u}, \hat{v}, \hat{p})=(U(y, t), 0,0)+\epsilon(u(y, t), v(y, t), p(y, t)) e^{i \alpha x}+\text { complex conjugate, }
$$

into (2.3) and (2.4), where $\epsilon \ll 1$ and $u, v, p=O(1)$. By eliminating the pressure $p$ and the streamwise velocity $u$ from the set of $O(\epsilon)$ equations we arrive at

$$
\frac{\partial}{\partial t}\left(\frac{\partial^{2} v}{\partial y^{2}}-\alpha^{2} v\right)+i \alpha U\left(\frac{\partial^{2} v}{\partial y^{2}}-\alpha^{2} v\right)-i \alpha \frac{\partial^{2} U}{\partial y^{2}} v=0
$$

where $\alpha$ is the wavenumber in the streamwise direction. For the case when the base flow, $U$, is independent of time we can write $v=\tilde{v}(y ; \alpha) e^{-i \omega t}$ and (2.6) reduces to the usual Rayleigh stability equation (1.1) where $\omega$ is the complex angular frequency of the disturbance wave. We shall henceforth refer to (2.6) as the time-dependent Rayleigh equation.

When the velocity of the jet is high, it is likely that the flow within the jet becomes turbulent. This turbulence could be due to the cavitation within the nozzle [36]. However, in this study we assume that any eddies which form within the jet are small so that our assumption that the jet can be approximated by the single velocity profile (2.2) still holds, along with the above linear approximation (2.6). In this formulation we assume that the main effect of the turbulent eddies is to modify the effective viscosity from molecular viscosity to some eddy viscosity, both of which are ignored in the inviscid theory.

In this study we wish to calculate the break-up length of the liquid jet, and this corresponds to calculating the distance from the nozzle to a point where the velocity component $\hat{v}$ becomes greater than some threshold value. Beyond this threshold value the disturbance can no longer be assumed to be a linear perturbation to the basic jet velocity and nonlinear effects become significant. We assume that this will be a precursor to rapid jet break up, and thus we assume that nonlinear saturation does not occur. We note at this point that the threshold for the onset of nonlinearity could also be given by $\hat{u}$, or perhaps even the perturbation kinetic energy, becoming larger than some threshold value. However, there is no evidence from experiments to suggest the use of one diagnostic over the other. Also, in this study we are interested in investigating the qualitative effect of unsteadiness on break-up length, and as all such diagnostics can be expressed in terms of $\hat{v}$, a different choice of diagnostic 
corresponds to just choosing a different threshold for $\hat{v}$. We do, however, acknowledge that to obtain quantitative agreement with experiments this issue will need to be investigated through experiments.

We calculate $\hat{v}$ by following the time evolution of each eigenmode of (2.6), which corresponds to a different value of $\alpha$, as the basic flow $U(y, t)$ changes with time. Once the time evolution of each eigenmode is known we can calculate the shape and amplitude of the wave packet as

$\hat{v}(x, y, t)=\epsilon \int_{0}^{\infty}\left(v(y, t ; \alpha) e^{i \alpha x}+\right.$ complex conjugate $) d \alpha=2 \epsilon \operatorname{Re}\left(\int_{0}^{\infty} v(y, t ; \alpha) e^{i \alpha x} d \alpha\right)$

where $\epsilon$ is the same as in (2.5). We assume that at $t=t_{0}$ a disturbance is forced at the nozzle, and the initial set of eigenmodes $v\left(y, t_{0} ; \alpha\right)$ are calculated by solving the Rayleigh equation using Newton iterations. Each of these eigenmodes is then integrated forward in time by solving (2.6) with homogeneous boundary conditions at $y= \pm \infty$. Due to the symmetry of the problem we need only consider the domain $y \in[0, \infty)$ with $v \rightarrow 0$ as $y \rightarrow \infty$ and

$$
v(0)=0 \quad \text { (varicose modes), }\left.\quad \frac{d v}{d y}\right|_{y=0}=0 \quad \text { (sinuous modes). }
$$

Turner et al. [22] showed that for steady planar jets at large velocities, it tends to be the sinuous modes that have the shortest break-up length and smallest break-up time. Therefore, in the current paper we only consider the evolution of sinuous modes, although the effect of unsteadiness is expected to be qualitatively similar for varicose modes. The initial amplitude of each eigenmode depends upon the forcing at the nozzle that generates the disturbance such that $v\left(y, t=t_{0} ; \alpha\right)=F(y, \alpha, \omega)$. In this paper we assume that

$$
v\left(y, t=t_{0} ; \alpha\right)=\tilde{v}(y ; \alpha)
$$

i.e. each eigenmode has a constant amplitude normalised such that $\tilde{v}(0 ; \alpha)=1$ for sinuous modes. The initial condition (2.8) corresponds to the forcing for each mode given by

$$
\hat{S}(x, y, t)=\frac{1}{4 \pi^{2}} \int_{F_{\alpha}} \int_{L_{\omega}} \tilde{v}(y ; \alpha) e^{i(\alpha x-\omega t)} d \omega d \alpha
$$

which is given in [21]. It is possible to consider different initial conditions such as

$$
v\left(y, t=t_{0} ; \alpha\right)=-\frac{i}{2 \pi D_{\omega}(\alpha, \omega)} \tilde{v}(y ; \alpha),
$$

with $\tilde{v}(0 ; \alpha)=1$ for sinuous modes, where $D(\alpha, \omega)=0$ is the dispersion relation $[37,38,21,22]$. This condition is equivalent to the forcing $\hat{v}(x, y=0, t)=$ $\delta(x) \delta\left(t-t_{0}\right)$ which forces each mode of the Rayleigh equation equally with unit amplitude. The reason we use (2.8) rather than (2.9) or any other initial 
condition is because a different initial condition will only make an algebraic difference to the break-up length calculation where the eigenmodes ultimately grow exponentially, and besides, we are interested in comparing the qualitative evolution of the jet in both an unsteady and quasi-steady manner which is independent of the choice of $F(y, \alpha, \omega)$. This is because the unsteady and quasi-steady calculations only differ by variations in the growth rates of the individual disturbance waves, not in the form of $F(y, \alpha, \omega)$, which is the same for both calculations. Results for different forcings can be obtained from a convolution integral using this impulse response, i.e. Green's functions. A true nozzle, such as a Diesel injector, may not typically force the eigenmodes as in (2.8) or (2.9), but might favour particular frequencies. Experimentally obtaining this frequency spectrum of eigenvalue amplitudes in the fluid is difficult and as yet has not been cleanly achieved. If quantitative agreement with experiments is required then the choice of forcing, and its effects should be fully investigated.

For the case when the parameter $q=\rho_{2} / \rho_{1}=1$, both the eigenmode and its derivative are continuous at the interface $y=1$, so we don't need any extra boundary conditions. However if $q \neq 1$ then we need to ensure that both the fluid velocity and the pressure are continuous across the interface (because our velocity profile is continuous across the interface). This gives the two supplementary equations

$$
\begin{aligned}
v_{1} & =v_{2} \\
\frac{\partial^{2} v_{1}}{\partial t \partial y}+i \alpha U \frac{\partial v_{1}}{\partial y}-i \alpha \frac{\partial U}{\partial y} v_{1} & =q\left(\frac{\partial^{2} v_{2}}{\partial t \partial y}+i \alpha U \frac{\partial v_{2}}{\partial y}-i \alpha \frac{\partial U}{\partial y} v_{2}\right)+W \alpha^{4} \eta(x, t)
\end{aligned}
$$

at $y=1$, where $W=W e^{-1}=\sigma /\left(\rho_{1} U_{0}^{* 2} L^{*}\right)$ is the inverse Weber number of the jet, $\sigma$ is the dimensional surface tension, and $\eta$ is the elevation of the free surface which satisfies $v_{1}=D \eta / D t$, where $D / D t$ is the convective derivative. Our motivation for this work comes from Diesel jets where typically $W e=O\left(10^{5}\right)$, therefore throughout this paper we neglect the effect of surface tension. However, at very low velocities the effect of surface tension becomes more significant, but this effect is not considered here. In the case of a steady basic flow, where $v_{1,2}=\tilde{v}_{1,2}(y ; \alpha) e^{-i \omega t}$, the condition $(2.11)$, with $W=0$, reduces to

$$
\rho_{1}(\alpha U-\omega) \frac{d \tilde{v}_{1}}{d y}-\rho_{1} \alpha \frac{d U}{d y} \tilde{v}_{1}=\rho_{2}(\alpha U-\omega) \frac{d \tilde{v}_{2}}{d y}-\rho_{2} \alpha \frac{d U}{d y} \tilde{v}_{2}
$$

which is the usual steady jump condition for pressure continuity at the fluid interface [15].

To find the unsteady evolution of the jet, we solve (2.6) directly, which we henceforth refer to as method (i). The numerical scheme used to solve (2.6) 
first maps the domain $y \in[0, \infty)$ to the domain $\bar{y} \in[0,1]$ using the mapping

$$
\bar{y}=\tanh (y) .
$$

The $\bar{y}$ direction is then discretized using finite differences with 10001 points for the case $q=1$ and 100001 points for $q \neq 1$, while a Crank-Nicolson approach is used to time step (2.6) with a time step $\delta_{t}=5 \times 10^{-3}$. This procedure continues for all wavenumbers which will experience growth over the evolution of the jet. For wavenumbers where $\omega_{i} \leq 0$ at $t=t_{0}$ we assume that the eigenmodes are initially neutral. This is a valid assumption because in the piecewise linear limit of the profile $(2.2)(\Delta \rightarrow 0)$ it can be shown that when the eigenmodes are not growing they are neutral, and hence for a profile close to the $\Delta=0$ limit we expect these waves to be approximately neutral. As we are interested in the maximum wave packet envelope of (2.7) we calculate

$$
|\hat{v}(x, y, t)|=2 \epsilon V\left(t_{0}\right)\left|\int_{0}^{\infty} v(y, t ; \alpha) e^{i \alpha x} d \alpha\right|,
$$

where this integral is calculated using the trapezoidal rule, with steps of $\delta_{\alpha}=5 \times 10^{-3}$. Here we multiply the wave packet amplitude by $V\left(t_{0}\right)=V_{0}$, because we assume that as the jet velocity increases the initial amplitude of the eigenmodes will also increase. This assumption does not affect the overall stability calculation because our analysis is linear, it only affects the break-up time and break-up length calculations in $\S 3$. This factor could be removed by making $\epsilon=\epsilon\left(V_{0}\right)$ but since we do not have any experimental evidence of how the eigenmode amplitudes behave, we believe this assumption is satisfactory.

Although the initial eigenmode for each $\alpha$ comes from an accurate Newton iteration of the Rayleigh equation, there tends to be an initial transient when solving (2.6) by the numerical method described above, where this initial condition adjusts to the finite difference mesh. Therefore, so as not to contaminate the break-up results, we run the unsteady code for 1001 iterations with $\delta_{t}=10^{-2}$ and the velocity profile fixed at $U\left(y, t_{0}\right)$ so as to allow the eigenmode to iterate to the correct steady result, before allowing the velocity profile to evolve in time. The function $|\hat{v}(x, y, t)|$ is then maximized over the $y$-direction to give the maximum amplitude of the wave packet at time $t$ as a function of distance $x$ from the nozzle.

There is very little experimental data giving the break-up length of a jet as it is being injected, but the experiments of [4] and [5] give steady jet break-up lengths for axisymmetric jets of the order of 80 to 300 nozzle radii, so in $\S 3$ we fix our break-up time (by fixing the parameter $\epsilon$ ) so that we have break-up lengths of this approximate order. Although we will have numerical timing restrictions stopping us from having too long a break-up length, this won't affect the unsteady/quasi-steady jet comparison in this paper.

So far we have considered calculating the time evolution of the eigenmodes 
of this problem by directly solving the partial differential equation (2.6). Another method to solve (2.6) is to assume that at each time step the jet profile changes, but the unsteady effect is neglected. Hence we solve Rayleigh's equation at each time step for a given value of $\alpha$, which generates the time evolution of the eigenmode and its growth rate, both of which can then be integrated via the trapezoidal rule. This approach is a quasi-steady approach, and in this study we shall examine when this approach approximates the unsteady solution well, and when the unsteady effects are important which makes these solutions significantly different. We henceforth refer to this quasi-steady method as method (ii). It is expected that the quasi-steady results will agree well with the unsteady results in regions of weak unsteady effects such as weak acceleration and when break-up times are short. For these cases the difference in growth rate caused by the unsteady effects don't have enough time to significantly change the break-up length. The quasi-steady result is useful because it is easier to calculate than the unsteady result, and, except for $q=1$, the iterative nature of the numerical scheme for the quasi-steady simulation makes it computationally less expensive than the unsteady simulations, because there is no discontinuity in gradient of the eigenmode across the interface.

The final approach we look at is when $U\left(y, t_{0}\right)$ is assumed to be steady at all future times, so

$$
v(y, t)=\tilde{v}(y ; \alpha) e^{-i \omega\left(t-t_{0}\right)} \quad t>t_{0} .
$$

This steady result is expected to agree well with the unsteady and quasisteady results only when acceleration effects are very small. We henceforth refer to this steady method as method (iii).

\section{Results}

In this paper we are interested in calculating the break-up length of an accelerating liquid jet and determining when the effect of acceleration is important. We are also interested in determining when the unsteady effect is important in the overall growth of the wave packet, and how this difference behaves as the jet profile evolves. The CFD results in figure 2 show how the velocity profiles vary throughout the acceleration period. From these axisymmetric profiles we can estimate the parameters $\delta_{1}, \delta_{2}$ and $\beta$ and these estimates are shown in figure $6(\mathrm{a})$. The parameter $\beta$ indicates the velocity value at the fluid interface, $\delta_{2}$ is chosen as the value where the velocity in the gas has decreased to approximately zero, and $\delta_{1}$ is found from the point at which the fluid velocity is $99 \%$ of the maximum velocity. This is approximately given by the dashed line in figure 2(b). At low velocities the fluid is bulging out of the nozzle and so has a slightly different behaviour due to the nozzle being full of fluid initially. 
This effect can be seen slightly in figure 2(a) where the fluid interface for results $1-3$ lies to the right of the vertical dashed line, at the discontinuity in gradient of the velocity profile. However, at the streamwise position where these profiles are observed, the bulging effect is less than is observed at the nozzle. We can approximate the behaviour of the parameters in figure 6(a) by using the piecewise approximations plotted by the solid lines. These fits are not ideal, but the purpose of this investigation is to examine the qualitative effect of acceleration on liquid jets, not to get quantitative agreement with experiments or CFD simulations. Therefore these approximations are adequate for our purposes. They are given by the following functions

$$
\begin{gathered}
\beta(V)=\left\{\begin{array}{l}
0.9-0.8 V^{1 / 2} \quad V<0.2722 \\
0.3+0.35 V^{1 / 2} \quad V \geq 0.2722
\end{array},\right. \\
\delta_{1}(V)=\left\{\begin{array}{l}
1.25 V^{1 / 2} \quad V<0.3054 \\
1.05-0.65 V^{1 / 2} V \geq 0.3054
\end{array},\right. \\
\delta_{2}(V)=0.26+e^{-9 V},
\end{gathered}
$$

where $V=V(t)$ is the jet centre-line velocity. These functions agree with the behaviour seen in figure 2, such that at moderate values of $V$ they give a velocity profile with weak shear layers in the inner and outer fluids, while when $V(t)=1$ (cf the larger velocity profiles in figure 2 ) the profiles have a thinner shear layer in the jet, and a larger value of $\beta$. An evolution of the velocity profile (2.2) as $V$ increases is shown in figure $6(\mathrm{~b})$ for $V=0.3,0.6,0.8,0.9$ and 0.99 and $\Delta=0.025$, where $\Delta$ is the smoothing parameter from (2.2). These are the initial velocity values considered in $\S 3.1$.

(a)

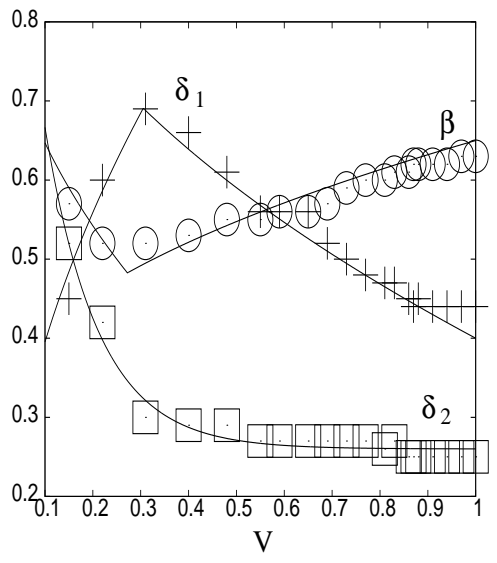

(b)

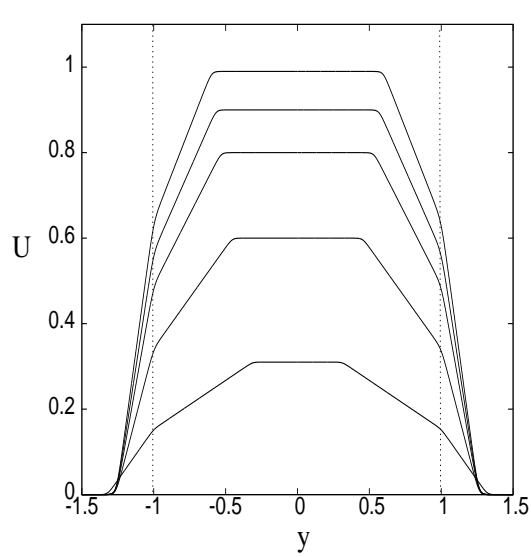

Fig. 6. The symbols in (a) plot the parameters $\delta_{1}, \delta_{2}$ and $\beta$ as functions of $V$ from the CFD simulations described in $\S 2$. The solid lines give the approximations (3.1)-(3.3). Panel (b) plots the jet velocity profile (2.2) for an accelerating jet with $V=0.3,0.6,0.8,0.9$ and 0.99 where the parameters $\delta_{1}, \delta_{2}$ and $\beta$ are given by (3.1) $-(3.3)$ and $\Delta=0.025$. 
In figures 1 and 3(b), we observed that the injection velocity for a typical jet injection in Diesel engines can be approximated by

$$
V(t)=\tanh (a t)
$$

where $a$ is a measure of the acceleration of the jet and $d V / d t=a\left(1-V^{2}\right)$. Typical values of the parameter $a$ from experiments lie roughly in the range $a \in[0.0005,0.006][30,31,6]$. Hence in this study we consider three different values of $a=10^{-2}, 10^{-3}$ and $10^{-4}$, which cover the orders of magnitude seen in Diesel jet experiments. From the experimental data shown in figure 1 , $a \approx 0.006$ while the CFD simulation in figure $3(\mathrm{~b})$ has $a \approx 0.002$.

If we consider the five profiles from figure $6(\mathrm{~b})$ in the limit of a steady jet (method (iii)), then each of these profiles has a growth rate $\omega_{i}$ for each real streamwise wavenumber $\alpha$ which can be calculated by solving the Rayleigh equation (1.1). These growth rates for the cases $q=1 / 35$ and $q=1$ are plotted in figure 7 . We consider the value $q=1 / 35$ as a representative value

(a)

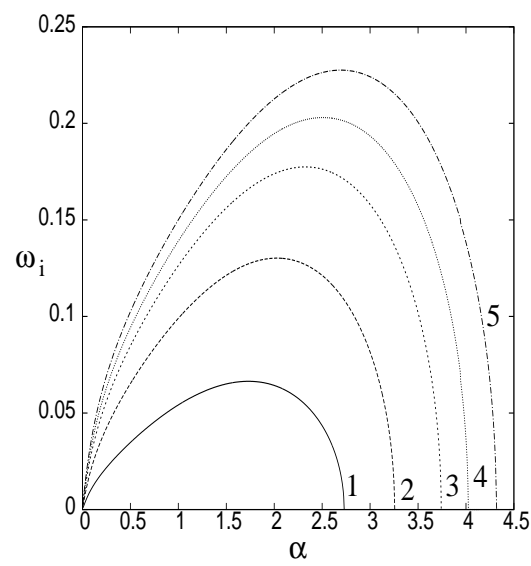

(b)

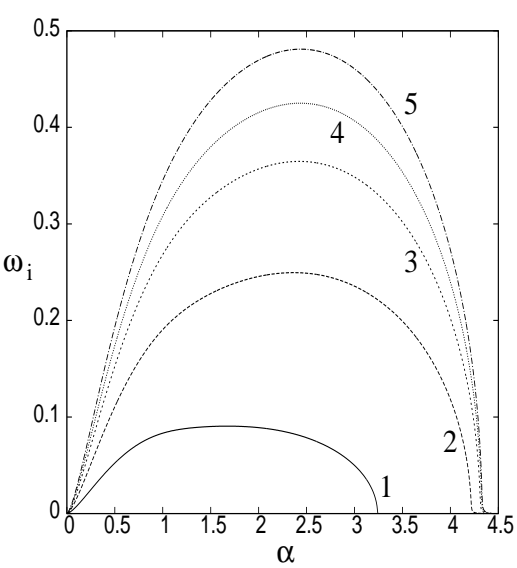

Fig. 7. Plot of the growth rate $\omega_{i}$ from $(2.13)$ as a function of the streamwise wavenumber $\alpha$ for method (iii) when (a) $q=1 / 35$ and (b) $q=1$. Both panels plot the growth rate at each of the velocities $V=0.3,0.6,0.8,0.9$ and 0.99 , numbered $1-5$ respectively.

because the range of values of $q$ in Diesel jet experiments typically lies between $q \in[1 / 60,1 / 10]$ depending upon the ambient gas density, the fuel involved and the temperature of injection [30,31,6]. Smaller values of $q$ are considered in experiments, but the qualitative behaviour of the wave packets for these density ratios is similar to the one considered here. We note at this point that the functions (3.1)-(3.3) would also be functions of $q$, but for simplicity this is neglected as results are expected to be similar for different $q$. For the experiment in figure $1 q \approx 1 / 12$ while for the CFD simulation in figure 3(b) $q \approx 1 / 40$.

The growth rates for $q=1 / 35$ in figure 7 (a) show that as $V$ is increased, the maximum value of $\omega_{i}$ increases (as expected because for a fixed profile 
$\left.\omega_{i}(V)=V \omega_{i}(V=1)\right)$, and the value of $\alpha$ at which this maximum occurs also increases with $V$. This suggests that the wavelength of the most unstable wave $(\lambda=2 \pi / \alpha)$ decreases as the jet accelerates. For $q=1$ in figure $7(\mathrm{~b})$ we see a similar behaviour of the growth rate except here the wavelength of the most unstable wave has a smaller increase at larger velocities.

In the next section, we examine how this variation of the growth rate as a function of velocity affects the break-up length of the jet. However, we first clarify how we calculate the jet break-up length. In this paper we assume that when the ratio of the amplitude of the generated disturbance compared to the basic flow, $|\hat{v}| / V$ reaches some critical threshold value then this is an indication that the perturbation is no longer small, which is a precursor to rapid break-up. As we have no experimental evidence for what this threshold value is, we choose $|\hat{v}| / V=0.2$ at break-up, where varying this value varies the break-up length and time (cf the earlier discussion in $\S 2$ ). From (2.5) we note that we also have the linearization parameter $\epsilon$ to define, but rather than vary this value we could absorb it into the above threshold criteria and say that break-up occurs when $V\left(t_{0}\right) V^{-1} \int_{0}^{\infty} v e^{i \alpha x} d x=2 \times 10^{5}$ times its initial amplitude. This is equivalent to $\epsilon=5 \times 10^{-7}$, which gives break-up lengths which are comparable to experiments.

\subsection{Break-up length calculations}

In this section we examine the break-up length of a jet where an individual disturbance is generated at the nozzle at times which correspond to the jet initially having the velocities $V=0.3,0.6,0.8,0.9$ and 0.99 which were considered earlier in this section. We start by examining how the wave packet evolves in space and time so that we can clearly define the break-up length of the wave packet. The case $q=1 / 35$ is numerically more challenging than the $q=1$ case, as well as computationally more expensive due to the presence of the fluid interface. Although the value of $q=1 / 35$ lies in the middle of the typical density ratios seen in Diesel jet experiments, we expect the results in this section to be qualitatively similar for different values of $q$ and as the main aim of this study is to get a qualitative understanding of transient jet break up, we do not consider other values here. We also acknowledge here that the parameters given in (3.1)-(3.3) probably do not have the correct behaviour for the case $q=1$, but we assume that they do for the purposes of developing the method in this paper. We believe this is justified as our ultimate goal is to understand the effect of acceleration on heavy jets injecting into lighter fluids $(q \lesssim 1 / 10)$.

Figure 8 shows a typical evolution of a wave packet for (a) $q=1 / 35$ and (b) $q=1$. Each panel shows the wave packet evolution for a steady disturbance 


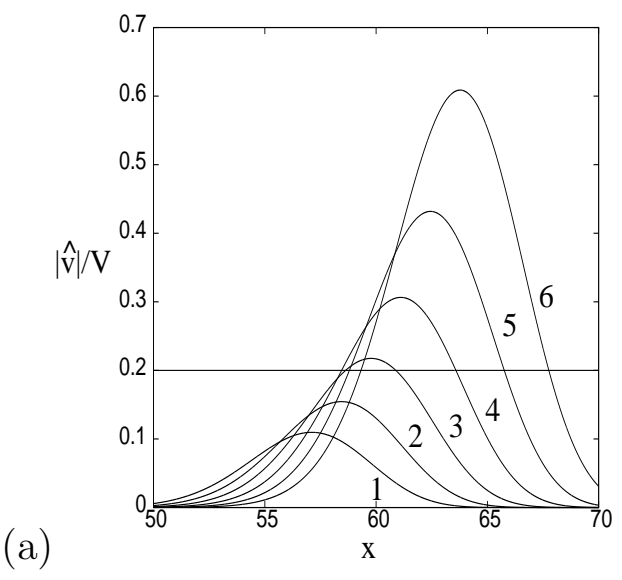

(b)

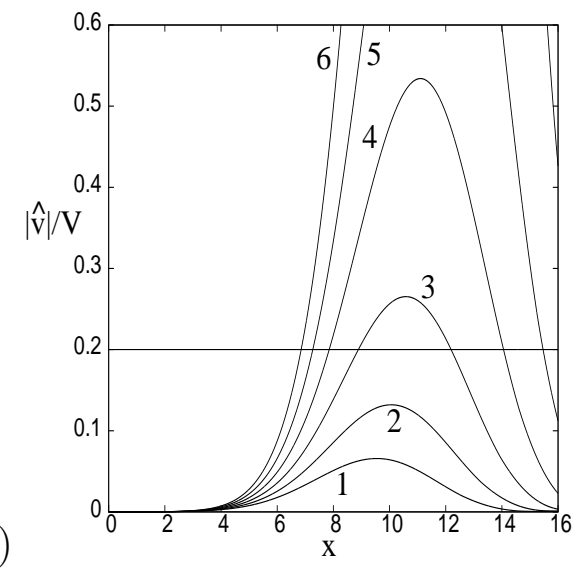

Fig. 8. Plot of the wave packet amplitude $|\hat{v}| / V$ as a function of $x$ for the steady result (method (iii) where $\left.V(t)=V\left(t_{0}\right)\right)$ with $(\mathrm{a})\left(V_{0}, q\right)=(0.8,1 / 35)$ and $(\mathrm{b})(0.8,1)$. In panel (a) the different lines correspond to the different time values $t-t_{0}=86$, 88, 90, 92, 94 and 96 numbered from $1-6$ respectively, while in panel (b) they correspond to $38,40,42,44,46$ and 48 respectively. The break-up length of this disturbance $x_{b}(t)$ is given by the left most point where the wave packet crosses the line $|\hat{v}| / V=0.2$.

(method (iii)) generated at $t=t_{0}$, where $V\left(t_{0}\right)=V_{0}=0.8$, as a function of the downstream distance, $x$. The horizontal line depicts the value $|\hat{v}| / V=0.2$ and we denote the break-up length of these disturbances to be the smallest value of $x$ for which $|\hat{v}| / V=0.2$. As the wave packet evolves the break-up length changes, and so we denote the break-up length by $x_{b}(t)$. For both cases we see that as the time after injection increases, the peak of the wave packet is convected downstream, and for $q=1 / 35$, we can see that once the jet reaches break up, the break-up length will first move upstream (see results 3 and 4 ), reach some minimum value $x_{b}^{\min }$, and then move downstream. The $q=1$ case in panel (b) appears to be different, as the break-up length only moves upstream for the time values considered. However, by considering the spatio-temporal stability analysis of [22], we know that in the piecewiselinear limit of this velocity profile $(\Delta \rightarrow 0)$, the wave packet is convectively unstable and this is also true for all the values of $V_{0}$ that we consider. Thus, we know that the trailing edge of the wave packet should eventually move downstream so that there is not any growth at $x=0$ which would give an absolute instability. However, we cannot numerically integrate the wave packet evolution for long enough to see this, because we are limited by giving the amplitude of each of our eigenmodes to 16 significant figures. Therefore, as some wavenumbers in the integral (2.12) grow much faster, and thus reach larger magnitudes than others, then machine error creeps into the integral due to the numerical round off error of each individual term of the integrand. This factor affects the results more for this value of $q$, because the wave packets are slowly convective requiring a very long time evolution, and this leads to some very large eigenmode magnitudes. This problem is less of an issue for the $q=1 / 35$ result except when we consider disturbances released with very 
small $V_{0}$, that also need a long time integration.

For the remainder of this section we consider the break-up length $x_{b}\left(t-t_{0}\right)$ for a particular disturbance generated at $t=t_{0}$, where $V\left(t_{0}\right)=V_{0}$ in (3.4). We examine the break-up lengths for different values of $a$ and $V_{0}$ for both the unsteady (i) and the quasi-steady (ii) methods.

(a)

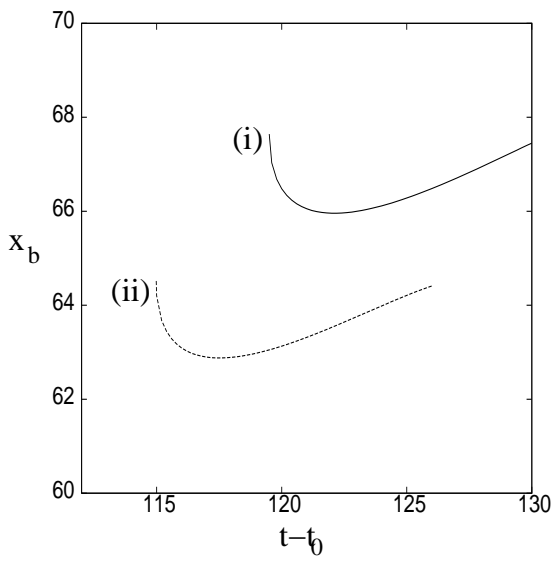

(b)

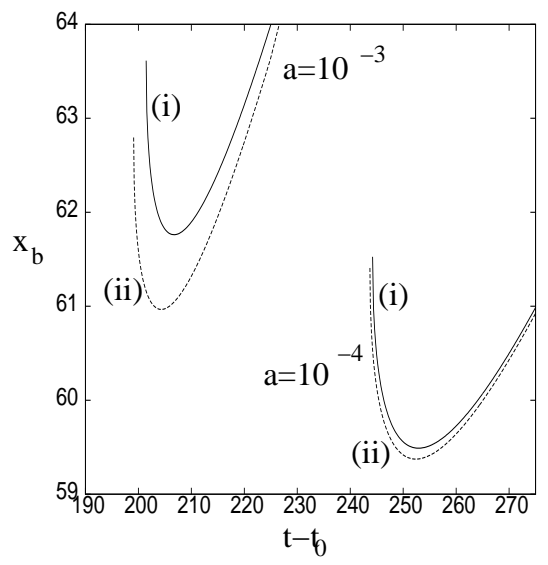

Fig. 9. Plot of the break-up length $x_{b}\left(t-t_{0}\right)$ for the case (a) $\left(V_{0}, a\right)=\left(0.3,10^{-2}\right)$ and (b) $\left(0.3,10^{-3}\right)$ and $\left(0.3,10^{-4}\right)$, all with $q=1 / 35$. The labels (i) and (ii) refer to the unsteady and quasi-steady solution methods respectively.

In figure 9 (a) we see that for $\left(V_{0}, a\right)=\left(0.3,10^{-2}\right)$ the effect of the acceleration has a significant effect on the break-up length of the jet. The effect of the acceleration (see method (i)) acts to increase both the break-up length and the initial break-up time of the jet when compared to the quasi-steady result (method (ii)). We shall see throughout this section that this effect is common for all values of $V_{0}$ and $a$ that we consider, but for this particular result we cannot say that the jet is quasi-steady. Here the overall minimum break-up length has increased by approximately 5\%, but this is still some way off the $50 \%$ maximum difference seen between some CFD simulations and experiments. When $a$ is decreased to $10^{-3}$ and $10^{-4}$ in figure $9(\mathrm{~b})$ we see that although the effect of the acceleration is still noticable, the jet can to all intents and purposes be regarded as quasi-steady, as the difference between the unsteady and quasi-steady results is greatly reduced. In fact for $a=10^{-3}$ the minimum break-up length has only increased by approximately $1 \%$. However, these results do show that even when acceleration effects are included in the break-up length calculation, $x_{b}(t)$ still has the same structure as the steady result, with $x_{b}$ decreasing from its initial value before being convected downstream.

In figure 10 we consider the break-up length of disturbances which leave the nozzle at later times with $V_{0}=0.6$ in figure 10 (a) and $V_{0}=0.8,0.9$ and 0.99 in figure $10(\mathrm{~b})$. For $\left(V_{0}, a\right)=\left(0.6,10^{-2}\right)$ we see that the effect of the acceleration is less than it was for $V_{0}=0.3$ with $x_{b}^{\text {min }}$ only increasing by approximately $3 \%$ in panel (a). As $a$ is again reduced the unsteady and quasi-steady results agree 
(a)

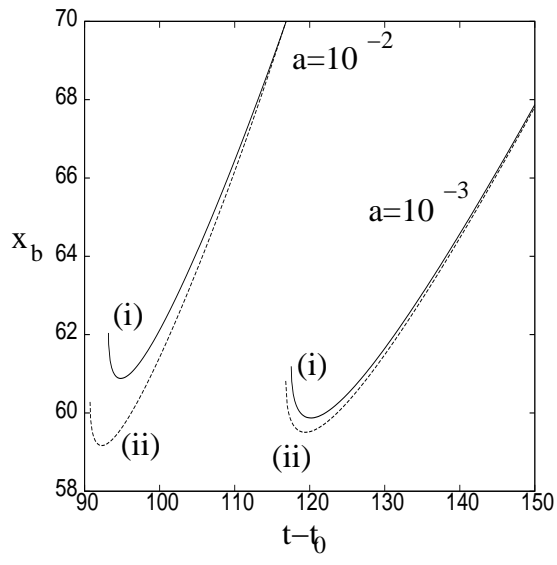

(b)

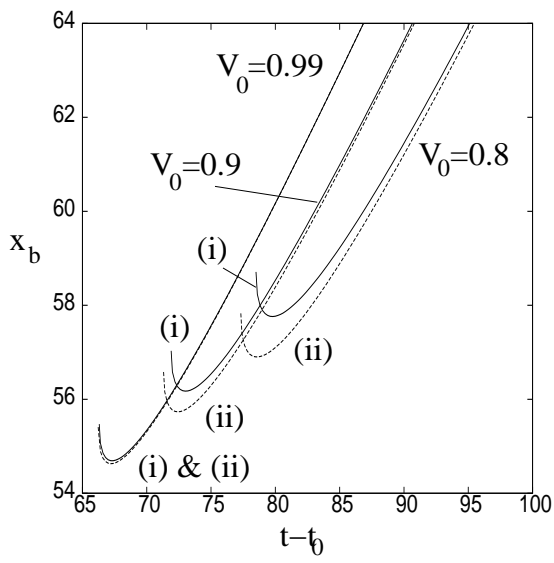

Fig. 10. Plot of the break-up length $x_{b}\left(t-t_{0}\right)$ for the case (a) $\left(V_{0}, a\right)=\left(0.6,10^{-2}\right)$ and $\left(0.6,10^{-3}\right)$ and $(\mathrm{b})\left(0.8,10^{-2}\right),\left(0.9,10^{-2}\right)$ and $\left(0.99,10^{-2}\right)$, all with $q=1 / 35$.

better and when $a=10^{-4}$ the results are indistinguishable (not shown). For $a=10^{-2}$ we can see in panel (b) that the effect of acceleration on the breakup length dramatically reduces as $V_{0}$ increases until the two results coincide for $V_{0}=0.99$. For smaller values of $a$ the unsteady and quasi-steady results practically coinside at these $V_{0}$ values.

(a)

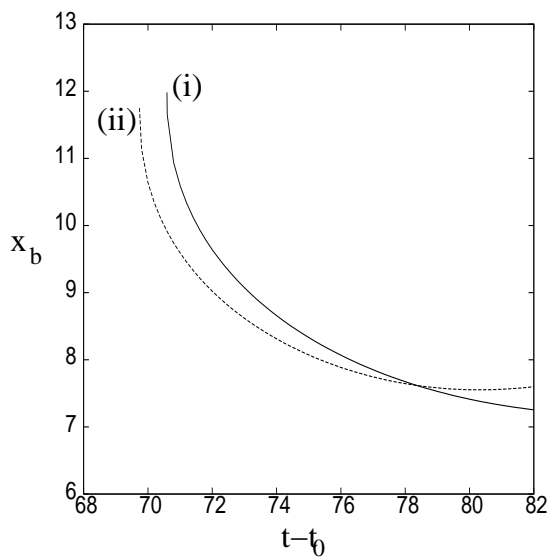

(b)

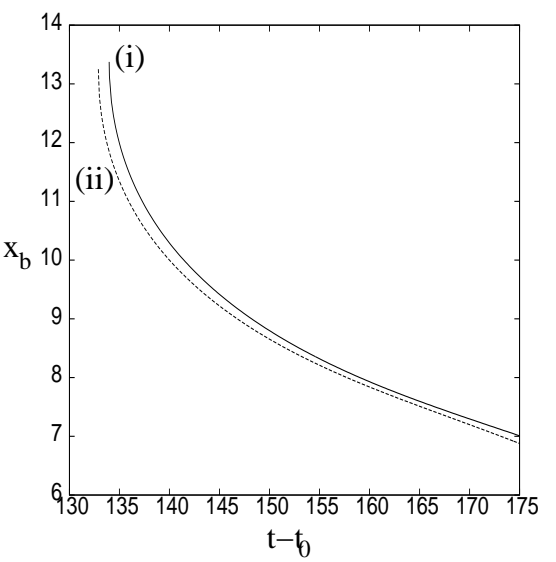

(c)

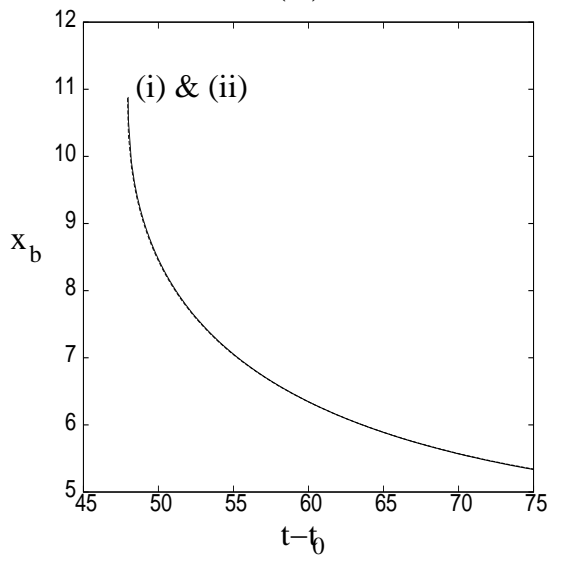

Fig. 11. Plot of the break-up length $x_{b}\left(t-t_{0}\right)$ for the case (a) $\left(V_{0}, a\right)=\left(0.3,10^{-2}\right)$ (b) $\left(0.3,10^{-3}\right)$ and (c) $\left(0.6,10^{-2}\right)$, all with $q=1$. 
For similar calculations with $q=1$, we find that the unsteady effect is reduced. We again find that the $\left(V_{0}, a\right)=\left(0.3,10^{-2}\right)$ result in figure 11 (a) is most affected by the acceleration, but not by nearly as much as the $q=1 / 35$ result in figure $9(\mathrm{a})$. The $\left(0.3,10^{-3}\right)$ result in figure $11(\mathrm{~b})$ has slight differences between the unsteady and the quasi-steady results, but for both smaller values of $a$, and larger $V_{0}$, see $\left(0.6,10^{-2}\right)$ in figure $11(\mathrm{c})$, we find both the unsteady and quasi-steady results coincide.

\subsection{Comparison of wave packet growth rates}

The results in $\S 3.1$ show how the jet acceleration affects the break-up length of a jet during its evolution. That is, to give an unsteady break-up length which is longer than that of the quasi-steady result. In this section we examine the growth rate of the wave packet, and the individual disturbance waves, to see how they are affected and change during the acceleration phase. This will show whether the wave packet for the unsteady result grows faster or slower than for the quasi-steady result, and how, more importantly, this depends upon the evolution of the velocity profile. To do this we examine the maximum value of the wave packet as a function of time. This will tell us how the peak of the wave packet is growing, and we assume that the trailing edge of the wave packet, which gives the break-up length of the jet, will behave in a similar manner.

We calculate the growth rate of the wave packet by taking a central finite difference time derivative of the log of the maximum wave packet amplitude, and we call this quantity $\lambda_{u, q}$, where the subscript $u$ or $q$ corresponds to the unsteady or quasi-steady result respectively. We then plot the ratio

$$
\Lambda=\frac{\lambda_{u}}{\lambda_{q}}
$$

as a function of $V$ for $\left(V_{0}, a\right)=\left(0.3,10^{-2}\right)$ and $\left(0.6,10^{-2}\right)$ in figure $12(\mathrm{a})$, which will be less than or greater than 1 depending upon which method gives the larger growth rate. Generating results for $V_{0}<0.3$ with $a=10^{-2}$ is numerically very expensive, due to the small time steps required to make the quasi-steady code converge. In this code the complex growth rate $\omega$ from (1.1) is found via Newton iterations at $t=t_{0}$. Then, $t$ is increased to the next time step $t=t_{0}+\delta_{t}$, and the initial guess for $\omega$ at this time step is the value of $\omega$ from the previous time step. Hence as $V_{0}$ reduces, the number of time-steps required for the code to converge increases dramatically due to the rapid variation of the growth rate curves as $V$ increases seen in figure 7(a). Therefore, because the solution of (1.1) has to be integrated out to large $y$ values for every iteration, the computation time increases rapidly. For smaller values of $a$, the jet centre-line velocity, and hence the velocity profile, changes 


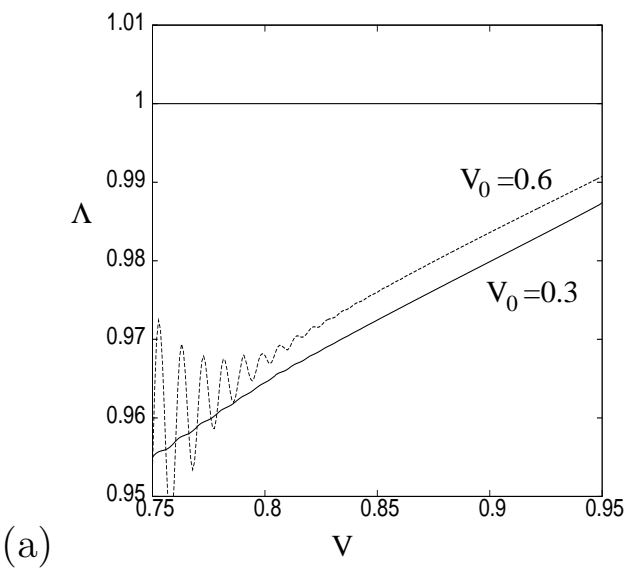

(b)

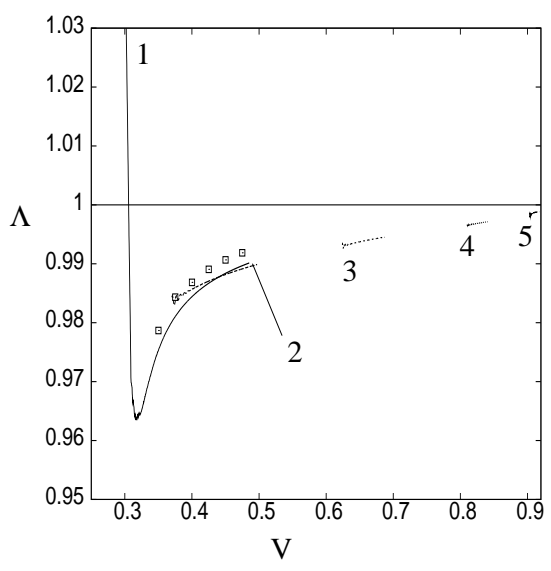

Fig. 12. Plot of $\Lambda(V)$ for (a) the cases $\left(V_{0}, a\right)=\left(0.3,10^{-2}\right)$ and $\left(0.6,10^{-2}\right)$ and (b) $\left(0.15,10^{-3}\right),\left(0.3,10^{-3}\right),\left(0.6,10^{-3}\right),\left(0.8,10^{-3}\right)$ and $\left(0.9,10^{-3}\right)$ with $q=1 / 35$ labelled $1-5$ respectively. The squares in panel (b) show the result for $\left(0.15,10^{-3}\right)$ with $\Delta=0.15$.

more slowly and thus this problem is removed. The results in this section also have an initial transient region where the wave packet is forming its distinctive shape seen in figure 8, before settling down to a smooth growth rate at large times (large $V$ ). This transient behaviour is a numerical issue and occurs because we have neglected all of the decaying eigenfunctions from the wave packet integral (2.12). However in this transient phase the magnitude of the wave packet is very small and so does not affect the results of $\S 3.1$. In this section we plot $\Lambda$ values after this transient phase has finished.

Once the wave packet has evolved, we see in figure 12(a) that $\Lambda$ is approximately the same for both wave packets at large $V$ with the slight difference being due to the initial eigenmode amplitudes of each individual wavenumber being different for each value of $V_{0}$. For example, when $V_{0}=0.6$, the eigenmodes initially have a uniform amplitude distribution, however when $V$ has accelerated to $V=0.6$ for the $V_{0}=0.3$ wave packet, the individual eigenmodes would have experienced some growth already so their amplitude distribution will not be uniform at $V=0.6$. Hence, the contribution of each eigenmode to the integral (2.12) at $V=0.6$ will be different for both the $V_{0}=0.3$ and $V_{0}=0.6$ wave packet, leading to different values of $\lambda_{u}$ and $\lambda_{q}$.

For $a=10^{-3}$ in figure $12(\mathrm{a})$ we note that $\Lambda<1$ which means the quasisteady wave packet will grow faster, and hence break up sooner, than the unsteady wave packet, as observed in $\S 3.1$. For the smaller acceleration value $a=10^{-3}$ in figure $12(\mathrm{~b})$ we were able to reduce the initial value of $V$ to $V_{0}=0.15$, which is the smallest value of $V$ for which we have a CFD data point in figure 6 (a). The qualitative behaviour seen for $V \in[0.15,0.3]$ in figure 12 (b) will also occur for the $a=10^{-2}$ result in panel (a), but of course the effect will be larger. These results show a similar behaviour to the $a=10^{-2}$ results, with the ratio $\Lambda<1$ and becoming closer to one as the acceleration 


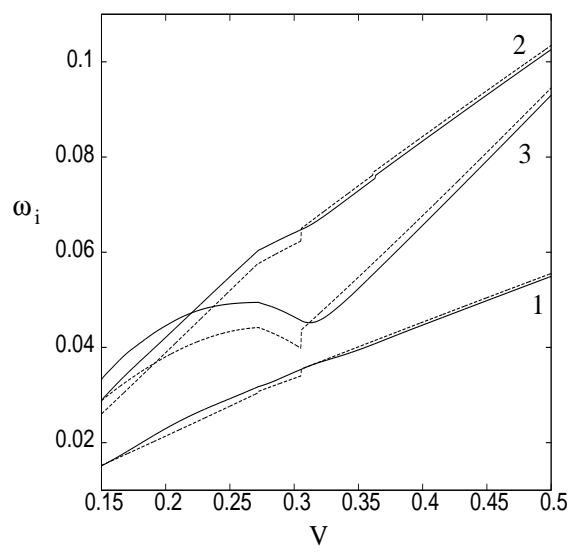

Fig. 13. Plot of the growth rate for the individual wavenumbers $\alpha=0.5,1.5$ and 2.5 , numbered 1-3 respectively, for the case $V_{0}=0.15$ and $a=10^{-3}$ in figure 12 . The unsteady growth rates are the solid lines and the quasi-steady growth rates are the dashed lines.

reduces. However, the $V_{0}=0.15$ result shows that for $V<0.3$ the value of $\Lambda>1$, implying that the growth rate of the unsteady wave packet is larger than that of the quasi-steady wave packet. This means that for disturbances released from the nozzle with $V_{0}<0.3$, the unsteady break-up length may not be longer than the corresponding quasi-steady break-up length. In fact it may even be shorter, but this depends upon the length of the break-up time. This can also be inferred from figure 13 which plots the growth rates for the individual wave numbers $\alpha=0.5,1.5$ and 2.5 from the $V_{0}=0.15$ result. Here the unsteady growth rates (solid lines) clearly change from being larger than the quasi-steady growth rates (dashed lines) to being smaller at around $V=0.3$, thus we can deduce that the unsteady effect when accompanied by thinning shear layers at the edge of the jet $(V \gtrsim 0.3054)$ slightly suppresses the growth rate and gives $\Lambda<1$, while for thickening shear layers $(V \lesssim 0.3054$ ) the acceleration amplifies the growth rate and gives $\Lambda>1$. This result, means that the $V_{0}=0.3$ results presented in $\S 3.1$ should give the maximum overestimate between the unsteady and the quasi-steady break-up lengths. Figure 13 also shows that the unsteady effect affects the growth rate of the shorter wavelength waves more than the longer waves.

Figure 12(b) also gives the result $\left(0.15,10^{-3}\right)$ with $\Delta=0.15$ in the velocity profile (2.2), given by the squares, which is compared to that for $\Delta=0.025$ given by the solid line. This shows that we expect similar results to those demonstrated in this paper to hold for smoother profiles. 


\subsection{Quasi-steady break-up model}

In order to get good agreement with the experimental jet break-up results, we should in principal use the full wave packet results of $\S 3.1$. However, because the wave packet is made up of the superposition of many individual disturbance waves, hundreds of hours of computational time goes into calculating $x_{b}(t)$ for a disturbance generated at a particular value of $V_{0}$. This calculation then needs to be repeated for a range of $V_{0}$ values to build up the structure of $x_{b}^{\min }(t)$. Most of the CFD codes that calculate jet penetration/breakup lengths used to date $[7,39,40,9,10]$ model the jet as a continuous string of droplets and only use the growth rate information of the fastest growing mode of the steady stability problem, in a quasi-steady manner, to calculate the break-up length of the jet in their simulation. (We note that an approach different from this one has been developed in stochastic spray models $[41,42]$ but the analysis of this approach is beyond the scope of this paper.) This is because this calculation is computationally faster than solving (2.6), which is desirable in CFD simulations because the fluid motion in $(x, y, t)$-space also needs to be solved at each time step, which itself is a time intensive calculation. Therefore, in this section we compare the wave packet break-up length results of $\S 3.1$ to break-up length calculations which are found by solely considering the fastest growing eigenmode of the disturbance. This is to investigate whether or not this method, which is similar to that used in CFD simulations, gives an accurate measure of the minimum break-up length of the wave packet or if this approximation also contributes to the break-up length discrepancy seen between experiments and CFD simulations. Firstly we consider a steady result to examine how close these approximate results are to the steady wave packet results (method (iii)), and then we investigate if the difference between these two results changes if we include the effect of acceleration.

In this section we consider only the growth of the eigenmode which has the largest growth rate at each time step of the numerical code. Therefore the approximation to this eigenmode is

$$
\hat{v}_{\text {approx }}(y, t)=\epsilon V_{0} \tilde{v}\left(y ; \alpha_{m}(V), \omega_{m}(V)\right) e^{-i \int_{t_{0}}^{t_{0}+t} \omega_{m}(V) d t},
$$

where $V_{0}=V\left(t_{0}\right), \omega_{m}$ is the maximum value of $\omega$ (see figure $7(\mathrm{~b})$ ) and $\alpha_{m}$ is the corresponding value of $\alpha$ at $\omega_{m}$. This is effectively the quasi-steady approach used in $§ 3.1$ except where only the fastest growing mode is considered, so we expect the results in this section with acceleration to approximate the quasi-steady results of $\S 3.1$. From $\S 3.1$ we know that using this quasi-steady approach is acceptable for calculating the break-up length except where the unsteady effects are significant, which occur for large accelerations and small values of $V_{0}$. We calculate the break-up length and break-up time of a disturbance generated at $t_{0}$, by finding the time value for which 
$\left|\hat{v}_{\text {approx }}(y, t) / V(t)\right|=2 \times 10^{5}$ from (3.5). This gives the time value at break-up, $t_{0}+t_{b}$, where $t_{b}$ is the break-up time, and then the corresponding approximate value of $x_{b}^{\min }\left(t_{b}\right)$ is found by solving

$$
\left.x_{b}^{\min }\left(t_{b}\right) \approx \int_{t_{0}}^{t_{0}+t_{b}} \frac{\partial \omega}{\partial \alpha}\right|_{\alpha=\alpha_{m}} d t
$$

The resulting points $\left(t_{b}, x_{b}^{\min }\left(t_{b}\right)\right)$ are shown as the squares in figure 14 and they can be compared to the respective result from $\S 3.1$ for the steady case, and the quasi-steady cases with $a=10^{-2}$ and $a=10^{-3}$.

(a)

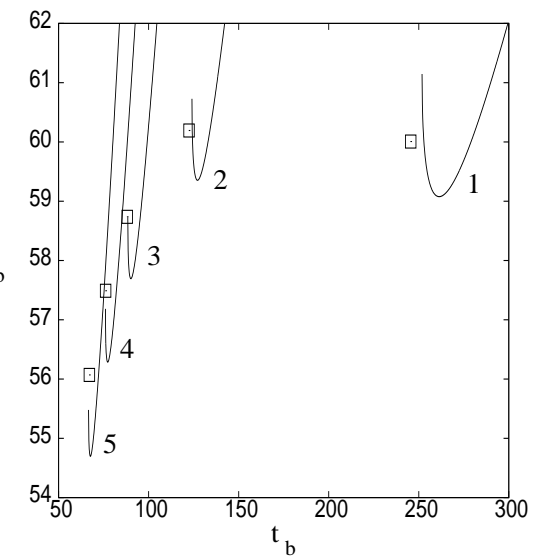

(c)

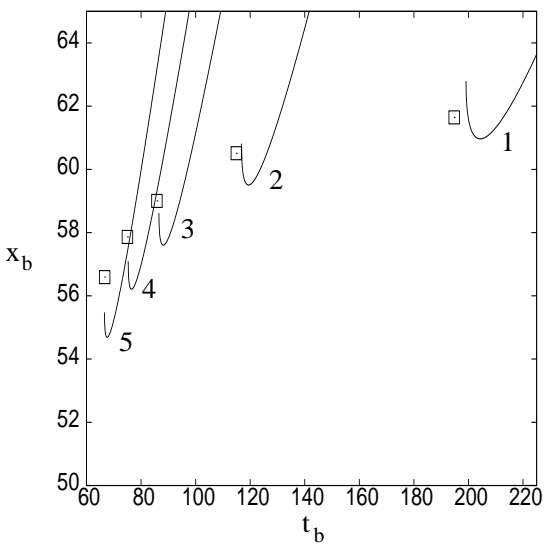

(b)

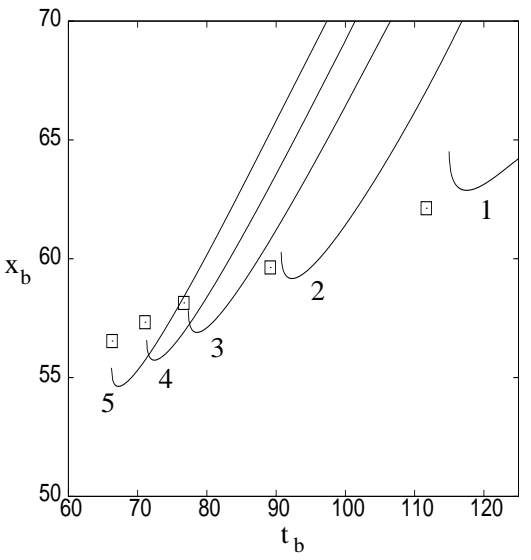

$(d)$

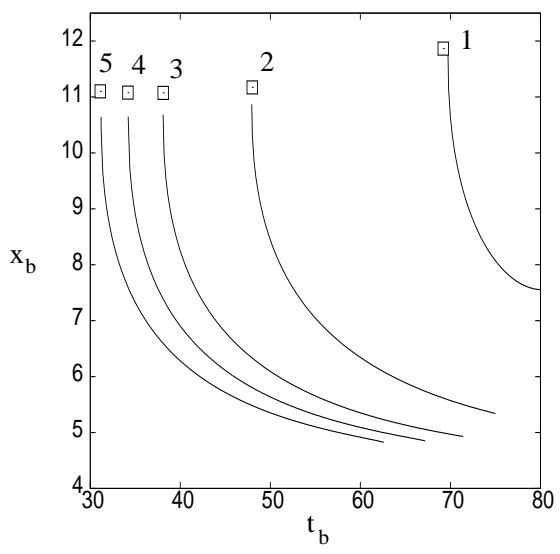

Fig. 14. Plot of the break-up length $x_{b}\left(t_{b}\right)$ from $\S 3.1$ comparing (a) the steady wave packet values $\left(V(t)=V_{0}\right)$ with the approximate initial break-up value $x_{b}^{\min }\left(t_{b}\right)$ and (b) and (c) the quasi-steady wave packet values with the approximate initial break-up value for $a=10^{-2}$ and $a=10^{-3}$ respectively. In each panel the lines 1-5 represent the disturbances with $V_{0}=0.3,0.6,0.8,0.9$ and 0.99 respectively, and the squares are from (3.6). Panel (d) plots the same results for the case $q=1$ and $a=10^{-2}$.

We observe in figure 14(a) that for the steady case the results of the approximate method (squares) are in reasonable agreement with the point of initial break-up of the jet. The results from this approximate method are in good agreement with the initial break-up time, and only over estimate the breakup length by about $2 \%$ for each result. Hence this method does a good job 
of calculating the break-up length for the steady case. When we consider the quasi-steady case with $a=10^{-2}$ in figure $14(\mathrm{~b})$ we see that for $V_{0}=0.8,0.9$ and 0.99 the results are similar to the steady results, with the approximate method again over-estimating the break-up length by approximately $2 \%$. But for $V_{0}=0.6$ and 0.3 the break-up length for the approximate method is now reduced in comparison with the quasi-steady wave packet result. For the $V_{0}=0.3$ result in particular the break-up length is now underestimated by approximately $1.5 \%$. When we combine this with the fact that the quasi-steady wave packet calculation is an underestimate of the unsteady break-up length by $5 \%$ then this could affect the results of the break-up problem but not by the amount seen in the CFD simulations. For $a=10^{-3}$, which is a value of $a$ more typical of Diesel jet experiments, in figure 14(c) we see that the effect of acceleration is greatly reduced and the approximate method is again in reasonably good agreement with the wave packet results. For the case $q=1$ in figure $14(\mathrm{~d})$ we see that this approximate method is poor for calculating the minimum break-up length here but does a good job of calculating the initial break-up length and time.

This appears to show that using the stability properties of steady jets in a quasi-steady manner should be acceptable for calculating the break-up length of the transient jets seen in Diesel jet experiments, at least to within a few percent.

\section{Conclusions and discussion}

In this paper we examined the effect of acceleration on the break-up characteristics of a liquid jet by examining the wave packet evolution of a velocity profile that behaves similarly to those calculated by CFD simulations. The results show that the unsteady effect is greatest where the acceleration is large. For $q=1 / 35$ we found that the unsteady effect of the acceleration caused the break-up length of the jet to increase compared to the quasi-steady result when the shear layers in the jet velocity profile were thinning, and we found the opposite effect when the shear layers of the velocity profile were thickening. For the largest acceleration parameter we considered, we found that the unsteady break-up length is approximately 5\% larger than the quasi-steady result for disturbances released at $t=t_{0}$ where the jet had initial velocity $V\left(t_{0}\right)=V_{0}=0.3$, but this percentage reduces for disturbances released at later times. However, this increase in break-up length is nowhere near the $50 \%$ difference seen between some experiments and CFD simulations [6]. These CFD simulations involve an approach which uses the stability properties of the fastest growing wave of steady jets, similar to the approximate method in $\S 3.3$, which we have found agree with our quasi-steady wave packet results to within a few percent. Thus this approximate quasi-steady approach should be 
adequate for calculating the break-up length of transient jets for parameter values that coincide with the Diesel jet experiments. However, there are other physical features of the injecting flow, such as the behaviour of the fluid at the leading edge of the jet that need to be considered before the full unsteady effect of acceleration can be neglected.

Therefore, we conclude that either a different mechanism for unsteadiness affects break up when $a<10^{-2}$ or the differences between the current CFD simulations and the experiments cannot be attributed to unsteady effects alone, if at all. If we compare our velocity profiles to those for steady jets at a similar density ratio in [22] we see that other than a larger value of $\delta_{2}$, the profiles are relatively similar. Therefore the accelerating jet profiles in this paper are very similar to those of a steady jet at the same velocity, so generating a quasi-steady result using the steady jet profiles would be almost identical to those generated in this paper. Therefore, unsteady effects may not be the cause for the difference between the CFD simulations and the experiments, but resolution of this discrepancy is beyond the scope of this paper.

In the present paper we studied the sinuous modes of a planar jet, as [22] showed that these modes tend to give the shortest break-up lengths in planar jets. However for axisymmetric jets the only unstable modes are varicose modes which give longer break-up lengths and break-up times than sinuous modes so this approach should be considered for axisymmetric jets if one wants to use the wave packet analysis to obtain quantitative agreement with experimental results. The axisymmetric case should also be checked to quantify if the $5 \%$ increase in break-up length found here for the planar jet case is comparable in magnitude to the increase for an axisymmetric jet. Another improvement to the current analysis would be to consider a forcing on the fluid similar to that exerted by a nozzle injecting the fluid. However, in this study we found that break-up length results of the initial amplitude condition (2.9), where the initial forcing was assumed to be a delta function in both space and time at $y=0$, were almost identical to those of (2.8), where all the eigenmodes were assumed to have equal amplitudes initially, as seen in figure 15, suggesting that this is not an important consideration. However, both these initial amplitude conditions assume that each wave number is forced to have a non-zero amplitude, but if for a true nozzle some of these amplitudes are zero then it could have a more significant effect on the break-up length results.

\section{Acknowledgments}

The authors would like to thank Cyril Crua and Elena Sazhina for supplying the experimental data used in this paper. This work is supported by the EPSRC under grants EP/F069855/1, EP/G000034/1 and EP/F058276/1. 


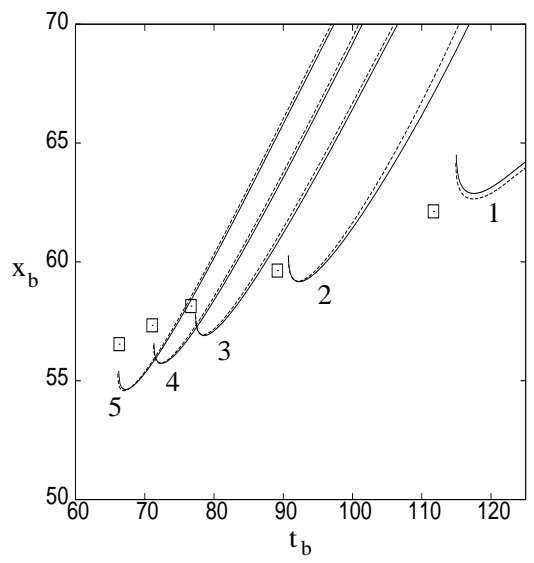

Fig. 15. Plot of the break-up length $x_{b}\left(t_{b}\right)$ from $\S 3.1$ comparing the quasi-steady wave packet values with the initial break-up value $x_{b}^{\min }\left(t_{b}\right)$ where the initial condition is given by (2.8) for the solid lines and (2.9) for the dashed lines. In each panel the lines $1-5$ represent the disturbances with $V_{0}=0.3,0.6,0.8,0.9$ and 0.99 respectively, and the squares are from (3.6).

\section{References}

[1] R. Stone, Introduction to internal combustion engines. MacMillan (New York), 1992.

[2] J. B. Heywood, Internal combustion engine fundamentals. McGraw-Hill (Singapore), 1998.

[3] S. S. Sazhin, "Advanced models for fuel droplet heating and evaporation," Progress in Energy and Combustion Science, vol. 32, no. 2, pp. 162-214, 2006.

[4] H. Hiroyasu, M. Shimizu, and M. Arai, "The break-up of high speed jet in a high pressure gaseous atmosphere," Proc. 2nd Int. Conf. on Liquid Atomization and Spray Systems, pp. 69-74, 1982.

[5] A. J. Yule and I. Filipovic, "On the break-up times and lengths of diesel sprays," Int. J. Heat and Fluid Flow, vol. 13, no. 2, pp. 197-206, 1992.

[6] S. S. Sazhin, S. B. Martynov, T. Kristyadi, C. Crua, and M. R. Heikal, "Diesel fuel spray penetration, heating, evaporation and ignition: modelling vs. experimentation," Int. J. Engineering Systems Modelling and Simulation, vol. 1, no. 1, pp. 1-19, 2008.

[7] R. D. Reitz, "Modelling atomization processes in high-pressure vaporizing sprays," Atomisation and Spray Technology, vol. 3, pp. 309-337, 1987.

[8] A. B. Liu, D. Mather, and R. D. Reitz, "Modeling the effects of drop drag and breakup on fuel sprays," SAE Report 930072, pp. 83-95, 1993.

[9] M. A. Patterson and R. D. Reitz, "Modelling the effects of fuel spray characteristics on diesel engine combustion and emission," SAE Paper 980131, 1998. 
[10] C. Chryssakis and D. N. Assanis, "A unified fuel spray breakup model for internal combustion engine applications," Atomization Sprays, vol. 18, pp. 1$52,2008$.

[11] L. D. Söderberg and P. H. Alfredsson, "Experimental and theoretical stability investigations of plane liquid jets," Eur. J. Mech B/Fluids, vol. 17, pp. 689-737, 1998.

[12] J. P. Kubitschek and P. D. Weidman, "Helical instability of a rotating viscous liquid jet," Phys. Fluids, vol. 19, p. 114108, 2007.

[13] J. P. Kubitschek and P. D. Weidman, "Helical instability of a rotating liquid jet," Phys. Fluids, vol. 20, p. 091104, 2008.

[14] N. Rimbert and G. Castanet, "Analysis of the Liquid Atomization out of a Full Cone Pressure Swirl Nozzle," ICMF 2010, Tampa, FL, USA, 2010.

[15] P. G. Drazin and W. H. Reid, Hydrodynamic Stability. Cambridge University Press, 1981.

[16] P. Marmottant and E. Villermaux, "On spray formation," J. Fluid Mech., vol. 498, pp. 73-111, 2004.

[17] S. J. Rees and M. P. Juniper, "The effect of confinement on the stability of viscous planar jets and wakes," J. Fluid Mech., vol. 656, pp. 309-336, 2010.

[18] Lord Rayleigh, The theory of sound, 2nd edn. Macmillian (London), 1894.

[19] T. Funada, D. D. Joseph, and S. Yamashita, "Stability of a liquid jet into incompressible gases and liquids," Int. J. Multiphase Flow, vol. 30, pp. 12791310, 2004.

[20] M. P. Juniper, "The effect of confinement on the stability of two-dimensional shear flows," J. Fluid Mech., vol. 565, pp. 171-195, 2006.

[21] M. P. Juniper, "The full impulse response of two-dimensional jet/wake flows and implications for confinement," J. Fluid Mech., vol. 590, pp. 163-185, 2007.

[22] M. R. Turner, J. J. Healey, S. S. Sazhin, and R. Piazzesi, "Stability analysis and break-up length calculations for steady planar liquid jets.," J. Fluid Mech., vol. 668 , pp. 384-411, 2011.

[23] P. Heurre and P. A. Monkewitz, "Local and global instabilities in spatially developing flows," A. Rev. Fluid Mech., vol. 22, pp. 473-537, 1990.

[24] S. F. Shen, "Some considerations on the laminar stability of time-dependent basic flows," J. Aerospace Sci., vol. 28, pp. 397-404, 417, 1961.

[25] G. Seminara and P. Hall, "Linear stability of slowly varying unsteady flows in a curved channel," Proc. R. Soc. Lond. A., vol. 346, pp. 279-303, 1975.

[26] S. H. Davis, "The stability of time-periodic flows," A. Rev. Fluid Mech., vol. 8, pp. 57-74, 1976. 
[27] P. Hall, "On the stability of the unsteady boundary layer on a cylinder oscillating transversely in a viscous fluid," J. Fluid Mech., vol. 146, pp. 347-367, 1984 .

[28] P. J. Blennerhassett and A. P. Bassom, "The linear stability of flat Stokes layers," J. Fluid Mech., vol. 464, pp. 393-410, 2002.

[29] P. Hall, "On the instability of Stokes layers at high Reynolds numbers," J. Fluid Mech., vol. 482, pp. 1-15, 2003.

[30] K. Karimi, E. M. Sazhina, W. A. Abdelghafffar, C. Crua, M. R. Heikal, and M. R. Gold, "Development in diesel spray characterisation and modelling," Proceedings of THIESEL-2006 Valencia, Spain, 2006.

[31] K. Karimi, "Characterisation of multiple-injection diesel sprays at elevated pressures and temperatures," PhD Thesis, University of Brighton, Brighton, United Kingdom, 2007.

[32] D. L. Youngs, Time-dependent multi-material flow with large fluid distortion. In Numerical methods for fluid dynamics (ed. K. W. Morton and M. J. Baines). Academic Press, 1982.

[33] J. H. Ferziger and F. M. Peric, Computational Methods for Fluid Dynamics, Third ed. Springer (Berlin), 2004.

[34] J. J. Healey, "Destabilizing effects of confinement on homogeneous mixing layers," J. Fluid Mech., vol. 623, pp. 241-271, 2009.

[35] H. B. Squire, "On the stability of three-dimensional disturbances of viscous flow between parallel walls," Proc. Roy. Soc. Lond. A, vol. 142, pp. 621-628, 1933 .

[36] C. Arcoumanis, M. Gavaises, H. Flora, and H. Roth, "Visualisation of cavitation in diesel engine injectors," Mec. Ind., vol. 2, pp. 375-381, 2001.

[37] P. J. Schmid and D. S. Henningson, Stability and transition in shear flows. Springer (New York), 2001.

[38] J. J. Healey, "A new convective instability of the rotating-disk boundary layer with growth normal to the disk," J. Fluid Mech., vol. 560, pp. 279-310, 2006.

[39] R. D. Reitz and R. Diwakar, "Structure of high-pressure fuel sprays," $S A E$ Paper 870598, 1987.

[40] P. J. O'Rourke and A. A. Amsden, "The Tab method for numerical calculation of spray droplet breakup," SAE Paper 872089, 1987.

[41] N. Rimbert and O. Séro-Guillaume, "Log-stable laws as asymptotic solutions to a fragmentation equation: Application to the distribution of droplets in a high Weber-number spray," Phys. Rev. E., vol. 69, p. 056316, 2004.

[42] M. Gorokhovski, J. Jouanguy, and A. Chtab, "LES with stochastic simulation of primary and secondary air-blast atomization," In Proc. ICLASS-2006, Kyoto, Japan, 2006. 